Da Silva, C.V., Martins, S.V., Villa, P.M., Correa Kruschewsky, G., Dias, A.A., Nabeta, F.H. (2021). Banco de semillas de relaves mineros como indicador de recuperación de vegetación en Brasil. Revista de Biología Tropical, 69(2), 700-716. https://doi.org/10.15517/rbt.v69i2.41800

\title{
Banco de semillas de relaves mineros como indicador de recuperación de vegetación en Mariana, Brasil
}

\author{
Carolina Viana Da Silva'; (D https://orcid.org/0000-0002-0300-8599 \\ Sebastião Venâncio Martins ${ }^{1 *}$; (D) https://orcid.org/0000-0002-4695-987X \\ Pedro Manuel Villa ${ }^{1 *}$; (D) https://orcid.org/0000-0003-4826-3187 \\ Gabriel Correa Kruschewsky²; (D) https://orcid.org/0000-0001-5911-2179 \\ Andreia Aparecida Dias ${ }^{2}$; (D) https://orcid.org/0000-0001-7257-1775 \\ Fabio Haruki Nabeta ${ }^{2}$; (D) https://orcid.org/0000-0002-1437-5503
}

1. Laboratório de Restauração Florestal, Departamento de Engenharia Florestal, Universidade Federal de Viçosa CEP 36570000, Viçosa, Minas Gerais, Brasil; carolviana777@gmail.com, venancio@ufv.br (Correspondencia*), pedro.villa@ufv.br (Correspondencia*)

2. Fundação Renova, Av. Getúlio Vargas, 671-Funcionários, CEP 30112-020, Belo Horizonte, Minas Gerais, Brasil; gabriel.kruschewsky@fundacaorenova.org, andreia.dias@fundacaorenova.org; fabio.nabeta@fundacaorenova.org

Recibido 13-V-2020. Corregido 13-IV-2021. Aceptado 04-VI-2021.

\section{ABSTRACT \\ Seed bank of mining tailings as indicator of vegetation regeneration in Mariana, Brazil}

Introduction: The soil seed bank is one of the most important ecological indicators for evaluating and monitoring the resilience of degraded ecosystems. Likewise, ecological restoration indicators are currently used in areas affected by the rupture of the Fundão tailings dam in Mariana, Minas Gerais, Brazil. Objective: To evaluate the seed bank at two depths of areas affected by mining tailings, Mariana municipality, Minas Gerais, Brazil. Methods: 15 samples $(40 \times 40 \mathrm{~cm})$ were collected per level of depth, in the upper layer $\left(\right.$ Depth1, $\mathrm{P}_{1}=0-2.5$ $\mathrm{cm}$ ), and lower layer (Depth2, $\mathrm{P}_{2}=2.5-5.0 \mathrm{~cm}$ ) of the mining tailings to survey the viable seed bank, which was later placed in a greenhouse for germination and seedling identification. The samples were placed in plastic trays to germinate in the nursery, then counted and identified weekly for six months. Results: The results showed that there is a seed bank in the surface layers of accumulated mining tailings in the affected areas of Mariana, with a marked difference between depths in terms of species richness and density of individuals. Higher species richness and number of individuals were observed in $\mathrm{P}_{1},(1165$ germinated seeds), belonging to 18 families and 47 species, in comparison to $\mathrm{P}_{2}$ (197 individuals), belonging to 12 families and 23 species. Probably the seed bank of layer $\mathrm{P}_{1}$ is influenced by the rain of seeds from the nearby forest. On the other hand, the $\mathrm{P}_{2}$ seed bank can be characteristic of mining tailings. Conclusions: Seed bank patterns can have positive effects on forest resilience at the local-scale, due to the high proportion of native, pioneer and anemochorical species of early successional stages.

Key words: disturbance; ecological indicators; regeneration strategy; species richness; dispersal syndrome. 
El banco de semillas del suelo es uno de los indicadores ecológicos más importantes para la evaluación y monitoreo de recuperación de comunidades vegetales en diferentes tipos de ecosistemas (Martins, Almeida, Fernandes, \& Ribeiro, 2008; Martins, 2009; Chazdon, 2014; Mall \& Singh, 2014). El banco de semillas del suelo consiste en la acumulación de propágulos viables que provienen de comunidades de plantas de áreas adyacentes (Chazdon, 2014; Mall \& Singh 2014; Martins, Borges, \& Silva, 2015). Las semillas permanecen latentes en el suelo por un tiempo determinado sin perder su viabilidad, hasta que ocurre algún tipo disturbio que induce su germinación, dando inicio a la regeneración natural (Martins, 2009; Chazdon, 2014; Mall \& Singh, 2014; Kildisheva et al., 2020). Existen varios atributos de los bancos de semillas que permiten evaluar su calidad y potencial para la regeneración natural o restauración activa de los ecosistemas (ej. transposición de banco), como por ejemplo, densidad de semillas viables, riqueza y composición de especies (Martins et al., 2015; Martins, 2018; Silva, Martins, Miranda-Neto, $\&$ Texeira, 2019). Sin embargo, estos atributos dependen de diferentes factores como la distancia de las fuentes de propágulos, presencia de dispersores, e intensidad en el uso de la tierra (Dalling, Swaine, \& Garwood, 1997; Martins \& Engel, 2007; Martins, 2018).

Después de la dispersión, algunas semillas quedan retenidas en la hojarasca, mientras que otras son depositadas a diferentes profundidades debido a sus características morfológicas (Baider, Tabarelli, \& Mantovani, 2001; Menezes, Neto, Azevedo, Machado, \& Nunes, 2019). Las semillas se pueden depositar a varias profundidades, dependiendo de la cantidad, tamaño y peso de las semillas, lo cual determina su período de viabilidad y capacidad de germinación (Menezes et al., 2019). La mayoría de los estudios indican una mayor densidad de semillas en las capas más superficiales del suelo donde prevalece un constante reemplazo de semillas (Baider et al., 2001; Martins et al., 2015). Así mismo, la mayor germinación de semillas ocurre en la capa más superficial del suelo, que puede estar fuertemente influenciada por las condiciones de luz y temperatura (Baider et al., 2001). Sin embargo, pocos estudios han demostrado estas variaciones de riqueza y composición de especies entre profundidades del banco de semillas.

El banco de semillas de bosques tropicales está compuesto principalmente por especies pioneras exigentes de luz, responsables de las primeras etapas de regeneración natural (Dalling et al., 1997; Martins, 2009; MirandaNeto \& Martins, 2017; Bechara et al., 2020). Sin embargo, la calidad y potencial del banco de semillas después de un disturbio es determinada por una alta riqueza de especies y variabilidad de la composición florística (Silva-Weber, Nogueira, Carpanezzi, Galvão, \& Weber, 2012; Balestrin, Martins, Schoorl, Lopes, \& Andrade, 2019). Por lo tanto, una alta densidad y riqueza de especies pioneras y tolerantes a la sombra de etapas avanzadas de sucesión en el mismo banco de semillas, es un indicador de alta calidad (Silva-Weber et al., 2012; Balestrin et al., 2019). Por esta razón, el banco de semillas ha sido considerado un importante indicador ecológico de degradación ambiental en diferentes escenarios de cambios en el uso de la tierra y disturbios antropogénicos, como por ejemplo la agricultura y minería como principales actividades de relevancia económica que afectan negativamente los ecosistemas (ej. Martins et al., 2008; Mall \& Singh, 2014; Miranda-Neto \& Martins, 2017). Así mismo, el banco de semillas permite evaluar el potencial de regeneración de áreas degradadas (Martins, 2018; Silva et al., 2019). Por otro lado, los ecosistemas de referencia sin disturbios pueden ser fuentes para la transposición de banco de semillas y nucleación durante la restauración activa (Martins, 2018; Silva et al., 2019). La nucleación es una técnica de restauración activa que consiste en el establecimiento y manejo de núcleos de plantas en áreas degradadas a partir de la transposición de bancos de semillas de ecosistemas conservados para promover la recuperación natural (Martins, 2018).

La minería es una de las principales actividades económicas de Brasil por parte del sector 
privado, que comprometen mayores esfuerzos e inversiones en actividades de mitigación ambiental y restauración ecológica (Silva et al., 2019; Martins, Villa, Balestrin, Nabeta, \& Silva, 2020). Por este motivo, las compañías mineras son responsables de la restauración de las áreas afectadas como procedimiento legal en Brasil (Martins et al., 2020). En este contexto, cabe resaltar que recientemente algunas áreas fueron afectadas por el impacto de los relaves mineros después de la ruptura de la represa de Fundão en Mariana, Minas Gerais, Brasil (Martins et al., 2020). Los relaves un conjunto de desechos de procesos mineros usualmente constituido por una mezcla de rocas molidas, agua y minerales (Campanharo et al., 2021). Por esta razón, las actividades de restauración ecológica han recibido alta prioridad en estas áreas afectadas mediante la aplicación combinada de diferentes métodos y técnicas de restauración activa y pasiva (Martins et al., 2020). Sin embargo, todavía existe la urgente necesidad de evaluar diferentes indicadores ecológicos que permitan identificar niveles de recuperación y ajustar métodos de restauración aplicados, como por ejemplo el monitoreo de bancos de semillas.

En este estudio se evaluó la variación de la riqueza, composición de especies, abundancia y parámetros fitosociológicos del banco de semillas a dos profundidades de relaves mineros en Mariana, municipio de Mariana, Minas Gerais, Brasil. Los principales parámetros fitosociológicos, fueron el número de individuos de todas las especies, frecuencia absoluta, frecuencia relativa, densidad absoluta y densidad relativa; así como grupos funcionales (estrategias de regeneración y síndromes de dispersión). Se tiene como premisa que este estudio es distinto de los trabajos tradicionales sobre el banco de semillas del suelo al comparar dos profundidades de relaves mineros. Por lo tanto, se establece como hipótesis que la profundidad del relave minero determina cambios en la distribución de la diversidad y estructura del banco de semillas debido a la diferenciación de grupos funcionales (estrategias de regeneración y síndromes dispersión). Específicamente se presume que existe una marcada variabilidad de la calidad del banco de semillas en términos de riqueza de especies, composición florística, abundancia y densidad de semillas entre profundidades del relave minero, a pesar de la poca diferencia de profundidad entre capas superficiales de relave (entre 0-2.5 cm, y entre $2.5-5 \mathrm{~cm}$ ). Como predicciones establecimos que el banco de semillas de la capa superior $\mathrm{P}_{1}(0-2.5 \mathrm{~cm})$ está dominado por especies pioneras y anemocóricas, e influenciado por la lluvia de semillas reciente desde los bosques cercanos. Por el contrario, el banco de semillas de la capa inferior $\mathrm{P}_{2}(2.5-5 \mathrm{~cm})$ puede presentar menor riqueza de especies y densidad de semillas viables, como resultado de relaves mineros acumulados.

\section{MATERIALES Y MÉTODOS}

Descripción del área de estudio: Descripción del área de estudio: El estudio se llevó a cabo entre julio y agosto de 2018 , en un área afectada por los relaves mineros resultantes de la ruptura de la represa de Fundão en el 2015 (Fig. 1A) en Brasil (Fig. 1B), Minas Gerais (Fig. 1C), específicamente en el municipio de Mariana $\left(23^{\circ} 02^{\prime} 70^{\prime \prime}-23^{\circ} 02^{\prime} 85^{\prime \prime} \mathrm{N}\right.$ \& $48^{\circ} 28^{\prime} 59^{\prime \prime}-48^{\circ} 28^{\prime} 63^{\prime \prime} \mathrm{W}$, Fig. 1D). El área de estudio se encuentra dentro de los límites de la Área Ambiental I de actuación de la Fundación Renova. Esta área corresponde a la zona de Minas Gerais, conocida como Quadrilátero Ferrífero dentro del bioma del Bosque Atlántico, con una altitud promedio de 712 metros. El clima de la región, según la clasificación de Köppen, es del tipo Cwa, con una precipitación promedio de $1307 \mathrm{~mm}$ y una temperatura promedio de $20.4{ }^{\circ} \mathrm{C}$ (Martins et al., 2020).

Los relaves acumulados en el área de estudio presentan diferentes profundidades (ca $80-100 \mathrm{~cm}$ ) sobre una topografía plana y homogénea a lo largo del río. La vegetación ribereña a lo largo del río Gualaxo do Norte se clasifica como bosque estacional semidecidual. Asimismo, cabe mencionar que el área de estudio tenía una larga historia de uso de suelo a base de pasto para el ganado antes de la acumulación de relaves mineros por el colapso de 
la presa de Fundão (Campanharo et al., 2020; Campanharo et al., 2021).

Muestreo del banco de semillas: Se establecieron 15 parcelas de $2 \times 2 \mathrm{~m}$ en área con relaves mineros en enero de 2019, completando un poco más de tres años después de la ruptura de la represa. Las parcelas fueron distribuidas bajo un diseño sistemático a lo largo de una hilera de $70 \mathrm{~m}$ con $5 \mathrm{~m}$ de separación en el área de relaves acumulados localizada entre dos fragmentos forestales separados por 250 $\mathrm{m}$ de distancia (Fig. 1). El banco de semillas fue muestreado a dos profundidades diferentes, una capa superior $\left(\mathrm{P}_{1}\right.$, hasta $\left.2.5 \mathrm{~cm}\right)$ y una capa inferior $\left(\mathrm{P}_{2}, 2.5-5.0 \mathrm{~cm}\right)$ del relave acumulado. Para recolectar el banco de semillas se usó un marco de tubería de PVC de $40 \times$ $40 \mathrm{~cm}$, que se arrojó aleatoriamente dos veces dentro de cada parcela, donde posteriormente fueron colectadas muestras de relaves para cada profundidad. Por lo tanto, se recolectó un total de 30 muestras de banco de semillas para cada profundidad.

Las 60 muestras de relaves se colocaron en bolsas de plástico transparentes debidamente etiquetadas y se enviaron para un vivero de la Universidad Federal de Viçosa, Viçosa, Minas Gerais. Las muestras fueron colocadas en bandejas plásticas de $0.25 \times 0.30 \times 0.05 \mathrm{~m}$ con orificios de drenaje y dispuestas sobre mesones de concreto de $1 \mathrm{~m}$ de altura en invernadero con un $50 \%$ de tela de sombra para evitar la contaminación externa. Las muestras de relave se sometieron a riego por aspersión programado (cuatro riegos diarios de tres minutos de duración) durante seis meses. El banco de semillas del suelo se evaluó a lo largo de este período con base en el método de emergencia de plántulas (ej. Silva et al., 2019). Las plántulas emergentes se contaron e identificaron una vez cada dos semanas e inmediatamente fueron retiradas

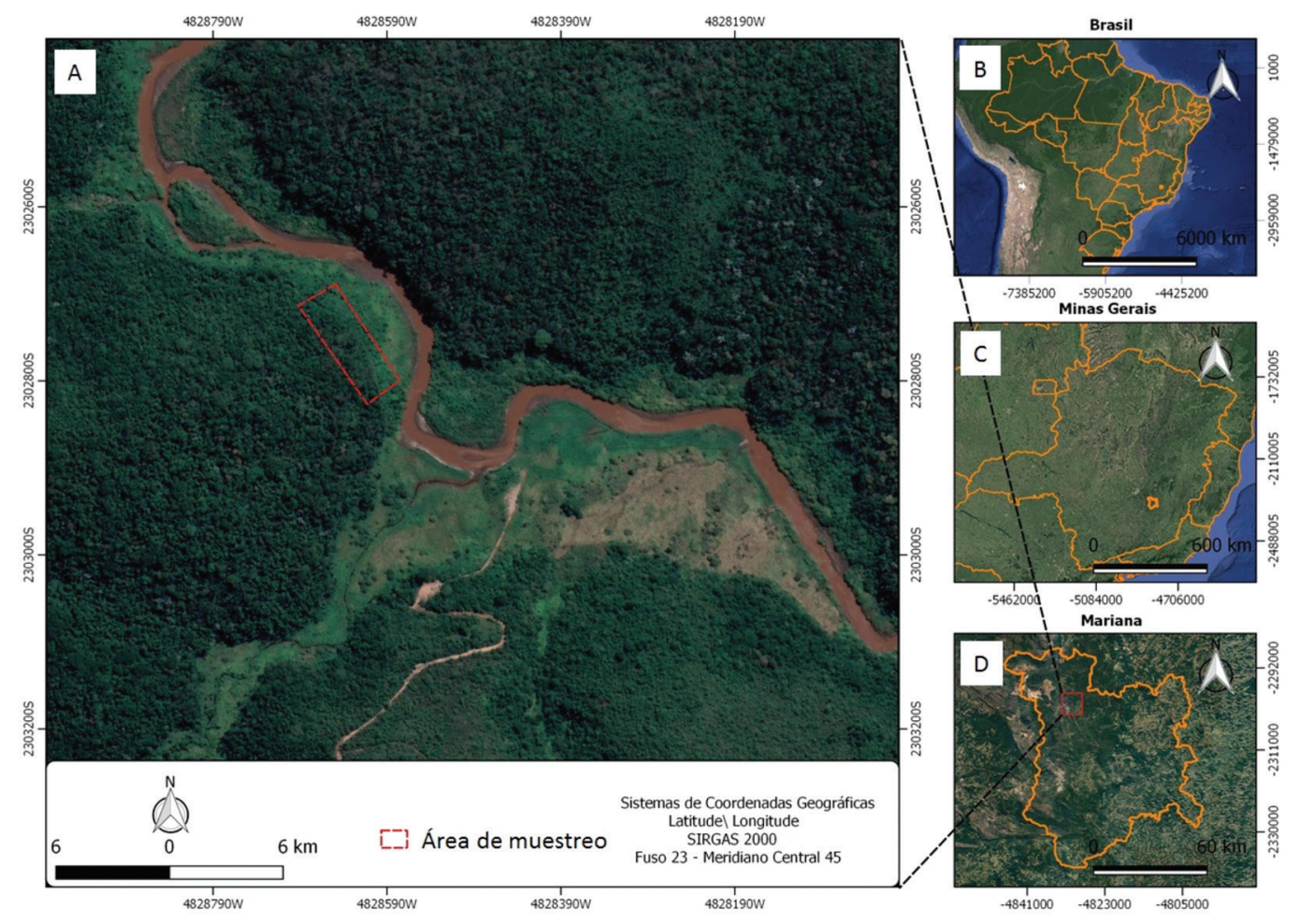

Fig. 1. Área de estudio en el río Gualaxo relación con A. América del Sur y B. Brasil, C. Minas Gerais y, D. Mariana. Fig. 1. Localization of the study area in the Gualaxo river according to A. South America B. Brasil, C. Minas Gerais and, D. Mariana. 
de las bandejas. Para corroborar que el banco de semillas no esté contaminado, se colocaron dos recipientes con arena gruesa esterilizada como un control, lo que permitirá registrar la germinación sólo de las semillas colectadas en el área de estudio (ej. Silva et al., 2019).

La identificación taxonómica de plantas germinadas se realizó de acuerdo con el sistema del Grupo de Filogenia Angiosperma (APG IV). Los nombres de las especies se verificaron utilizando la base de datos en el sitio web del Jardín Botánico de Missouri. Las plantas se identificaron a nivel de familia y especie. Las especies encontradas en los bancos de semillas se clasificaron, según la categoría sucesional o estrategia de regeneración, como especies: pioneras, secundarias iniciales, secundarias tardías. También se realizó una clasificación con respecto a las formas de vida (herbáceas, arbustos y árboles) y el síndrome de dispersión (autocóricas, anemocóricas y zoocóricas), además del origen de las especies (exóticas y nativas).

Análisis de datos: Para comparar la riqueza de especies entre los bancos de semillas de las dos profundidades se estimaron curvas de rarefacción y extrapolación basadas en el número de parcelas con el primer número de Hill, $q=0$ (Chao et al., 2014). Se hicieron extrapolaciones basadas en la presencia y ausencia de especies en las parcelas (Colwell et al., 2012). La rarefacción se estimó como la media de 100 repeticiones de bootstrapping para estimar intervalos de confianza del $95 \%$. Cuando los intervalos de confianza del $95 \%$ no se superponen, significa que los números de especies diferían significativamente en $\mathrm{P}<$ 0.05 (Colwell et al., 2012). Estas estimaciones se obtuvieron utilizando el paquete "iNEXT" (Hsieh, Ma, \& Chao, 2016) del software estadístico R (R Core Team, 2018). Para comparar la densidad de individuos de especies (datos no distribuidos normalmente) entre profundidades se usó la prueba de Wilcoxon para muestras pareadas $(\mathrm{P}<0.05)$. Para esta variable, se probó la distribución normal con la prueba de Shapiro-Wilk, evaluando la gráfica
Q-Q, y la homogeneidad de varianza mediante la prueba de Bartlett usando el programa $\mathrm{R}$ (R Core Team, 2018).

Realizamos un análisis de coordenadas principales (PCoA), basado en la matriz de disimilitud de Bray-Curtis para comparar los efectos de la profundidad sobre los patrones de composición de especies utilizando el paquete "vegan" en el programa R (R Core Team, 2018). Así mismo, utilizamos el análisis de varianza multivariado con permutaciones (PERMANOVA, 9999 permutaciones) para determinar las posibles diferencias en la composición de especies utilizando la rutina 'adonis', disponible en el mismo paquete "vegan" (R Core Team, 2018).

A partir del número de individuos de todas las especies (NI), se estimaron la frecuencia absoluta (FA), la frecuencia relativa (FR), la densidad absoluta (DA) y la densidad relativa (DR) de todas las especies muestreadas en los bancos de semillas. La frecuencia relativa de las especies se obtuvo de la frecuencia absoluta, dividiendo el número de parcelas en las que está presente la especie por el número total de parcelas. La densidad relativa se obtuvo a partir de la densidad absoluta calculada a partir del número total de especies presentes en una parcela dividida por el área total muestreada (Balestrin et al., 2019; Villa et al., 2019).

Las parcelas también fueron agrupadas con datos de abundancia de cada especie, a partir de una matriz de Bray-Curtis (disimilitud florística) usando el método de agrupamiento de medias aritméticas no ponderadas (UPGMA). De esta forma, se utilizó el índice de Sørensen para estimar el grado de similitud florística entre las parcelas. Todos los análisis se llevaron a cabo usando el programa $\mathrm{R}$ ( $\mathrm{R}$ Core Team, 2018), excepto para el análisis UPGMA que se utilizó el programa PC-Ord 5.14 (ej. Villa et al., 2019).

\section{RESULTADOS}

Flora del banco de semillas: Después de seis meses de monitoreo del banco de semillas, se registró un total de 1362 plántulas 
germinadas considerando ambas profundidades. Del total de plantas registradas, 1165 pertenecen a la capa superior $\left(\mathrm{P}_{1}\right)$, que corresponde a aproximadamente el $85.5 \%$ del total; mientras que la capa inferior $\left(\mathrm{P}_{2}\right)$ se observó un total de 197 plántulas germinadas. Del total de plántulas germinadas, 47 especies correspondieron a 18 familias en $\mathrm{P}_{1}$; mientras que en la capa inferior del relave $\left(\mathrm{P}_{2}\right)$, se registraron 12 familias y 23 especies.

Densidad y riqueza de especies: Considerando cada profundidad de relave muestreado, se observó un total de 485.4 individuos $\mathrm{m}^{2}$ en $\mathrm{P}_{1}$, y de 82.09 individuos $\mathrm{m}^{2}$ en $\mathrm{P}_{2}$. La familia que obtuvo la mayor densidad en número de individuos fue Cyperaceae, que equivale a 58.2 $\%$ y $60.4 \%$ del total, para $\mathrm{P}_{1}$ y $\mathrm{P}_{2}$ respectivamente. Cuando se compara la variabilidad del número de individuos se observa que existen diferencias significativas $(\mathrm{z}=1,23, \mathrm{P}<0.001)$ entre profundidades (Fig. 2A). La riqueza de especies también difirió significativamente $(\mathrm{P}$ $<0.05)$ entre las profundidades. La riqueza de especies en el banco de semillas de la capa
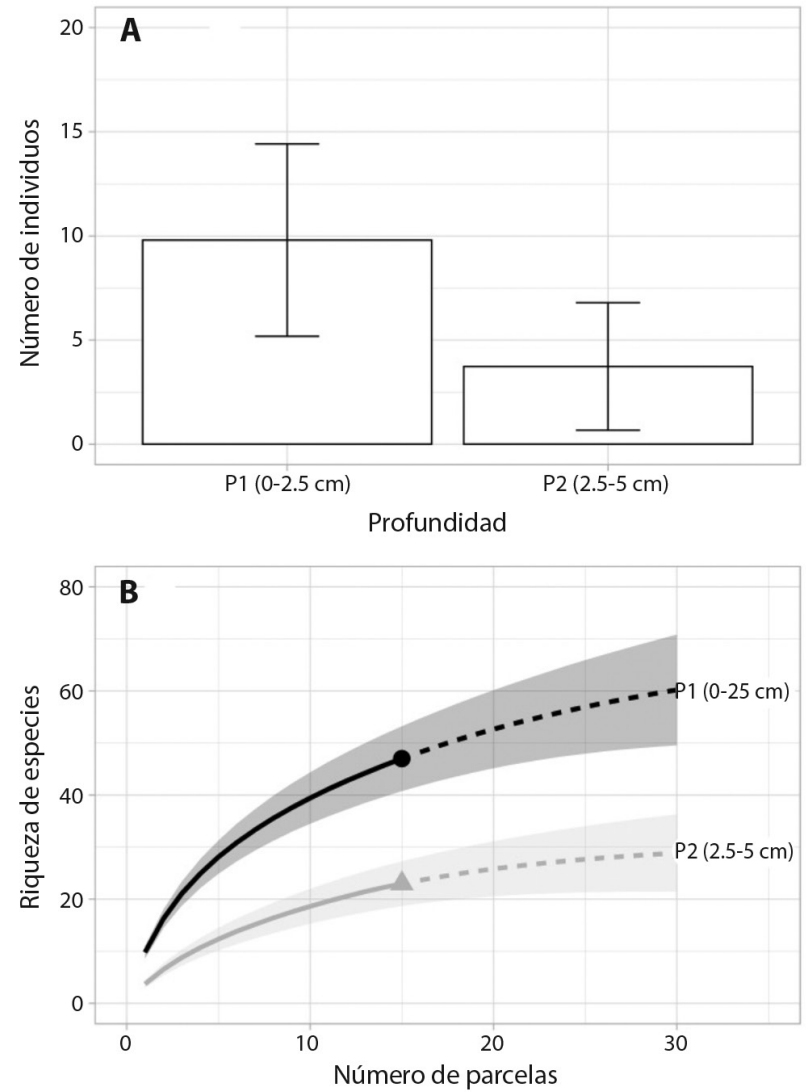

Fig. 2. A. Diferencias en la variación del número de individuos por parcela entre las dos profundidades $\left(\mathrm{P}_{1}\right.$ y $\left.\mathrm{P}_{2}\right)$ de relaves. B. Curvas de rarefacción (línea continua) y extrapolación (líneas discontinuas) de riqueza de especies basada en el número de muestras de dos profundidades $\left(\mathrm{P}_{1}\right.$ y $\left.\mathrm{P}_{2}\right)$ de relaves mineros en Mariana, Minas Gerais, Brasil. Las curvas de rarefacción y extrapolación presentan las líneas que representan los valores medios y las bandas la desviación estándar con intervalos de confianza del $95 \%$.

Fig. 2. A. Differences in the variation in the number of individuals per plot between the two tailings depths $\left(\mathrm{P}_{1}\right.$ and $\left.\mathrm{P}_{2}\right)$. B. Rarefaction curves (continuous line) and extrapolation (dashed lines) of species richness based on the number of samples from two depths $\left(\mathrm{P}_{1}\right.$ and $\left.\mathrm{P}_{2}\right)$ of mining tailings in Mariana, Minas Gerais, Brazil. The rarefaction and extrapolation curves present the lines that represent the mean values and the standard deviation bands with $95 \%$ confidence intervals. 
superior fue hasta más de $50 \%$ mayor que en la capa inferior (Fig. 2B).

\section{Composición florística y parámetros} fitosociológicos: Las familias con el mayor número de especies fueron Asteraceae (14 especies), Cyperaceae y Poaceae (cinco especies cada una) en ambas profundidades del relave. En la capa superior, se destacaron las familias Asteraceae, Solanaceae, Onagraceae y Lamiaceae, que representaron alrededor del 79 $\%$ del total (Tabla 1).

En la capa inferior, se destaca la presencia de las familias Solanaceae, Asteraceae y
Lamiaceae, consecutivamente, que representan el $47.11 \%$ del total de semillas emergentes (Tabla 2).

Banco de semillas por categoría de dispersión y regeneración: Con respecto al origen de las especies, se observó que sólo el $2.7 \%$ son de origen exótico y $97.3 \%$ nativas (Tabla 3). En relación con el grupo ecológico de regeneración, ambas profundidades presentaron un mayor número de individuos pioneros y secundarios. En $\mathrm{P}_{1}$, se encontraron $71 \%$ de los individuos pioneros y $14 \%$ de los secundarios iniciales; mientras que en $\mathrm{P}_{2}, 58$

TABLA 1. Parámetros fitosociológicos en la capa superior del relave $\left(\mathrm{P}_{1}=0-2.5 \mathrm{~cm}\right)$.

TABLE 1. Phytosociological parameters in the upper layer of the tailings $\left(\mathrm{P}_{1}=0-2.5 \mathrm{~cm}\right)$

\begin{tabular}{|c|c|c|c|c|c|}
\hline Nombre científico & NI & DA & DR & FA & FR \\
\hline Cyperus rotundus $\mathrm{L}$. & 365.0 & 2281.3 & 31.33 & 0.80 & 8.11 \\
\hline Digitaria sanguinalis (L.) Scop. & 218.0 & 1362.5 & 18.71 & 0.33 & 3.38 \\
\hline Cyperus difformis $\mathrm{L}$. & 168.0 & 1050.0 & 14.42 & 0.27 & 2.70 \\
\hline Hypolytrum pungens (Vahl) Kunth & 45.0 & 281.3 & 3.86 & 0.33 & 3.38 \\
\hline Solanum paniculatum $\mathrm{L}$. & 42.0 & 262.5 & 3.61 & 0.53 & 5.41 \\
\hline Cyperus distans L. f. & 34.0 & 212.5 & 2.92 & 0.40 & 4.05 \\
\hline Hyptis suaveolens (L.) Poit. & 27.0 & 168.8 & 2.32 & 0.20 & 2.03 \\
\hline Ludwigia tomentosa (Cambess.) H. Hara & 27.0 & 168.8 & 2.32 & 0.20 & 2.03 \\
\hline Solanum americanum Mill. & 26.0 & 162.5 & 2.23 & 0.67 & 6.76 \\
\hline Pluchea sagittalis (Lam.) Cabrera & 24.0 & 150.0 & 2.06 & 0.53 & 5.41 \\
\hline Erechtites hieraciifolius (L.) Raf. ex DC. & 21.0 & 131.3 & 1.80 & 0.60 & 6.08 \\
\hline Melastomataceae sp. & 17.0 & 106.3 & 1.46 & 0.13 & 1.35 \\
\hline Echinochloa colona (L.) Link & 16.0 & 100.0 & 1.37 & 0.13 & 1.35 \\
\hline Lepidium virginicum $\mathrm{L}$. & 16.0 & 100.0 & 1.37 & 0.47 & 4.73 \\
\hline Vernonia polyanthes (Spreng.) Less. & 16.0 & 100.0 & 1.37 & 0.67 & 6.76 \\
\hline Gnaphalium pensylvanicum Willd & 14.0 & 87.5 & 1.20 & 0.20 & 2.03 \\
\hline Eleusine indica (L.) Gaertn. & 10.0 & 62.5 & 0.86 & 0.33 & 3.38 \\
\hline Scoparia dulcis L. & 10.0 & 62.5 & 0.86 & 0.27 & 2.70 \\
\hline Asteraceae sp. & 8.0 & 50.0 & 0.69 & 0.13 & 1.35 \\
\hline Gnaphalium purpureum L. & 6.0 & 37.5 & 0.52 & 0.27 & 2.70 \\
\hline Acanthospermum australe (Loefl.) Kuntze & 5.0 & 31.3 & 0.43 & 0.07 & 0.68 \\
\hline Solanum mauritianum Scop & 5.0 & 31.3 & 0.43 & 0.20 & 2.03 \\
\hline Begonia cucullata Willd. & 4.0 & 25.0 & 0.34 & 0.07 & 0.68 \\
\hline Eclipta alba (L.) Hassk. & 4.0 & 25.0 & 0.34 & 0.07 & 0.68 \\
\hline Ixora gardneriana Benth & 4.0 & 25.0 & 0.34 & 0.13 & 1.35 \\
\hline Eleocharis elegans (Kunth) Roem. \& Schult. & 3.0 & 18.8 & 0.26 & 0.07 & 0.68 \\
\hline Mikania sp. & 3.0 & 18.8 & 0.26 & 0.20 & 2.03 \\
\hline Brachiaria plantaginea (Link.) Hitchc & 2.0 & 12.5 & 0.17 & 0.07 & 0.68 \\
\hline Clidemia hirta (L.) D. Don & 2.0 & 12.5 & 0.17 & 0.13 & 1.35 \\
\hline Conyza canadensis (L.) Cronquist & 2.0 & 12.5 & 0.17 & 0.13 & 1.35 \\
\hline
\end{tabular}




\begin{tabular}{lccccc}
\hline Ludwigia alternifolia L. & 2.0 & 12.5 & 0.17 & 0.07 & 0.68 \\
Mikania cordifolia (L.f.) Willd & 2.0 & 12.5 & 0.17 & 0.13 & 1.35 \\
Não identificada 1 & 2.0 & 12.5 & 0.17 & 0.13 & 1.35 \\
Phyllanthus tenellus Roxb. & 2.0 & 12.5 & 0.17 & 0.07 & 0.68 \\
Ageratum conyzoides L. & 1.0 & 6.3 & 0.09 & 0.07 & 0.68 \\
Cecropia hololeuca Miq. & 1.0 & 6.3 & 0.09 & 0.07 & 0.68 \\
Commelina diffusa Burm. f. & 1.0 & 6.3 & 0.09 & 0.07 & 0.68 \\
Erechtites valerianifolius (Link ex Spreng.) DC. & 1.0 & 6.3 & 0.09 & 0.07 & 0.68 \\
Glycine wightii (Graham ex Wight \& Arn.) Verdc. & 1.0 & 6.3 & 0.09 & 0.07 & 0.68 \\
Não identificada 2 & 1.0 & 6.3 & 0.09 & 0.07 & 0.68 \\
Paspalum paniculatum L. & 1.0 & 6.3 & 0.09 & 0.07 & 0.68 \\
Rubus rosifolius Sm. & 1.0 & 6.3 & 0.09 & 0.07 & 0.68 \\
Sida rhombifolia L. & 1.0 & 6.3 & 0.09 & 0.07 & 0.68 \\
Spermacoce latifolia Aubl. & 1.0 & 6.3 & 0.09 & 0.07 & 0.68 \\
Trema micrantha (L.) Blume & 1.0 & 6.3 & 0.09 & 0.07 & 0.68 \\
Triumfetta semitriloba Jacq. & 1.0 & 6.3 & 0.09 & 0.07 & 0.68 \\
Vernonanthura phosphorica (Vell.) H. Rob. & 1.0 & 6.3 & 0.09 & 0.07 & 0.68 \\
Total & 1165 & 7282.4 & 100.0 & 9.91 & 100.0 \\
\hline
\end{tabular}

Número de individuos de todas las especies (NI), frecuencia absoluta (FA), frecuencia relativa (FR), densidad absoluta (DA) y densidad relativa (DR).

Number of individuals of all species (NI), absolute frequency (FA), relative density (FR), absolute density (DA) and relative density (DR).

$\%$ pioneros y $23 \%$ secundarios iniciales. Del número total de individuos, dos especies se identificaron como secundarias tardías en $\mathrm{P}_{1}$, Ixora gardneriana y Rubus rosifolius; mientras que en $\mathrm{P}_{2}$ solo se registró Rubus rosifolius. Por otro lado, se observó que, del total de especies germinadas, el $89.35 \%$ correspondió a herbáceas, seguido por $7.05 \%$ arbustivas, $0.9 \%$ de especies arbóreas y $0.3 \%$ de trepadoras o lianas. Finalmente, con la clasificación de síndromes de dispersión se observó que el 84.12 $\%$ de las especies fueron anemocóricas, $9.87 \%$ zoocóricas y $3.09 \%$ autocóricas (Tabla 3).

Patrones de similitud florística: Los resultados demuestran una alta similitud florística entre las parcelas de las dos profundidades (Fig. 3). Por ejemplo, un grupo identificado con aproximadamente un $85 \%$ de similitud florística, principalmente de especies gramíneas, se encuentra entre las parcelas $\mathrm{P}_{3}, \mathrm{P}_{18}, \mathrm{P}_{19}$ y $\mathrm{P}_{13}$. El análisis de dendrograma bidireccional indicó que la vegetación en el área de estudio podría dividirse en seis grupos con parcelas compartidas entre profundidades, mostrándose una alta similitud florística (rango entre 70 y 90 \%) entre parcelas y grupos (Fig. 3).

Composición de especies: La composición de especies no tuvo diferencias significativas entre las dos profundidades (Permanova: $\mathrm{F}_{1,28}=2.72, \mathrm{P}=0.11$ ), corroborando la alta similitud florística observada (Fig. 4). A pesar de la alta variabilidad de la composición florística en el estrato inferior (P2) explicada principalmente por el $\mathrm{PCoA}_{1}(52 \%)$, existe una alta superposición de los dos grupos de profundidad en el espacio multivariante (Fig. 4).

\section{DISCUSIÓN}

Los resultados de este estudio demostraron que actualmente prevalece un banco de semillas viables compuesto principalmente por especies nativas en las capas superficiales de relaves mineros acumulados en Mariana. La presencia de un banco de semilla en relaves mineros estudiados representa un importante indicador de recuperación de comunidades 
TABLA 2. Parámetros fitosociológicos en la capa inferior del relave $\left(\mathrm{P}_{2}=2.5-5.0 \mathrm{~cm}\right)$

TABLE 2. Phytosociological parameters in the lower layer of the tailings $\left(\mathrm{P}_{2}=2.5-5.0 \mathrm{~cm}\right)$

\begin{tabular}{|c|c|c|c|c|c|}
\hline Nombre científico & NI & DA & DR & FA & FR \\
\hline Cyperus rotundus $\mathrm{L}$. & 79.00 & 493.75 & 40.10 & 0.60 & 16.07 \\
\hline Digitaria sanguinalis (L.) Scop. & 27.00 & 168.75 & 13.71 & 0.40 & 10.71 \\
\hline Cyperus difformis $\mathrm{L}$. & 23.00 & 143.75 & 11.68 & 0.33 & 8.93 \\
\hline Solanum americanum Mill. & 12.00 & 75.00 & 6.09 & 0.47 & 12.50 \\
\hline Cyperus distans L. $\mathrm{f}$. & 11.00 & 68.75 & 5.58 & 0.13 & 3.57 \\
\hline Hypolytrum pungens (Vahl) Kunth & 6.00 & 37.50 & 3.05 & 0.07 & 1.79 \\
\hline Hyptis suaveolens (L.) Poit. & 5.00 & 31.25 & 2.54 & 0.07 & 1.79 \\
\hline Pluchea sagittalis (Lam.) Cabrera & 5.00 & 31.25 & 2.54 & 0.07 & 1.79 \\
\hline Scoparia dulcis L. & 5.00 & 31.25 & 2.54 & 0.27 & 7.14 \\
\hline Echinochloa colona (L.) Link & 2.00 & 12.50 & 1.02 & 0.07 & 1.79 \\
\hline Eclipta alba (L.) Hassk. & 2.00 & 12.50 & 1.02 & 0.13 & 3.57 \\
\hline Eleusine indica (L.) Gaertn. & 2.00 & 12.50 & 1.02 & 0.13 & 3.57 \\
\hline Erechtites hieraciifolius (L.) Raf. ex DC. & 2.00 & 12.50 & 1.02 & 0.13 & 3.57 \\
\hline Ludwigia tomentosa (Cambess.) H. Hara & 2.00 & 12.50 & 1.02 & 0.07 & 1.79 \\
\hline Melastomataceae sp. & 2.00 & 12.50 & 1.02 & 0.13 & 3.57 \\
\hline Paspalum paniculatum $\mathrm{L}$. & 2.00 & 12.50 & 1.02 & 0.13 & 3.57 \\
\hline Rubus rosifolius $\mathrm{Sm}$. & 2.00 & 12.50 & 1.02 & 0.07 & 1.79 \\
\hline Sida rhombifolia $\mathrm{L}$. & 2.00 & 12.50 & 1.02 & 0.07 & 1.79 \\
\hline Solanum paniculatum $\mathrm{L}$. & 2.00 & 12.50 & 1.02 & 0.13 & 3.57 \\
\hline Glycine wightii (Graham ex Wight \& Arn.) Verdc. & 1.00 & 6.25 & 0.51 & 0.07 & 1.79 \\
\hline Gnaphalium pensylvanicum Willd & 1.00 & 6.25 & 0.51 & 0.07 & 1.79 \\
\hline Lepidium virginicum $\mathrm{L}$. & 1.00 & 6.25 & 0.51 & 0.07 & 1.79 \\
\hline Vernonia polyanthes (Spreng.) Less. & 1.00 & 6.25 & 0.51 & 0.07 & 1.79 \\
\hline Total & 197.0 & 1231.2 & 100.0 & 3.73 & 100.0 \\
\hline
\end{tabular}

Número de individuos de todas las especies (NI), frecuencia absoluta (FA), frecuencia relativa (FR), densidad absoluta (DA) y densidad relativa (DR).

Number of individuals of all species (NI), absolute frequency (FA), relative density (FR), absolute density (DA) and relative density (DR).

TABLA 3. Especies clasificadas de acuerdo a la estrategia de regeneración, síndrome de dispersión, origen y forma de vida

TABLE 3. Species classified according to regeneration strategies, dispersal syndrome, origin and forms of life

\begin{tabular}{|c|c|c|c|c|}
\hline Familias/Especies & ER & DISP & ORG & FV \\
\hline \multicolumn{5}{|l|}{ Asteraceae } \\
\hline Acanthospermum australe (Loefl.) Kuntze & $\mathrm{Nc}$ & Zoo & $\mathrm{N}$ & $\mathrm{H}$ \\
\hline Ageratum conyzoides L. & $\mathrm{Pi}$ & Zoo & $\mathrm{N}$ & $\mathrm{H}$ \\
\hline Asteraceae sp. & - & - & - & - \\
\hline Conyza canadensis (L.) Cronquist & $\mathrm{Pi}$ & Ane & $\mathrm{E}$ & $\mathrm{H}$ \\
\hline Eclipta alba (L.) Hassk. & $\mathrm{Si}$ & Ane & $\mathrm{N}$ & $\mathrm{H}$ \\
\hline Erechtites hieraciifolius (L.) Raf. ex DC. & $\mathrm{Pi}$ & Ane & $\mathrm{N}$ & $\mathrm{H}$ \\
\hline Gnaphalium pensylvanicum Willd & $\mathrm{Si}$ & Ane & $\mathrm{N}$ & $\mathrm{H}$ \\
\hline Gnaphalium purpureum L. & $\mathrm{Si}$ & Ane & $\mathrm{N}$ & $\mathrm{H}$ \\
\hline
\end{tabular}




\begin{tabular}{|c|c|c|c|c|}
\hline Mikania sp. & - & - & - & - \\
\hline Mikania cordifolia (L.f.) Willd & $\mathrm{Pi}$ & Ane & $\mathrm{N}$ & $\mathrm{T}$ \\
\hline Pluchea sagittalis (Lam.) Cabrera & $\mathrm{Nc}$ & Ane & $\mathrm{N}$ & $\mathrm{H}$ \\
\hline Vernonanthura phosphorica (Vell.) H. Rob. & $\mathrm{Pi}$ & Ane & $\mathrm{N}$ & $\mathrm{B}$ \\
\hline Vernonia polyanthes (Spreng.) Less. & $\mathrm{Pi}$ & Ane & $\mathrm{N}$ & $\mathrm{B}$ \\
\hline \multicolumn{5}{|l|}{ Begoniaceae } \\
\hline Begonia cucullata Willd. & $\mathrm{Pi}$ & Ane & $\mathrm{N}$ & $\mathrm{H}$ \\
\hline \multicolumn{5}{|l|}{ Brassicaceae } \\
\hline Lepidium virginicum $\mathrm{L}$. & $\mathrm{Pi}$ & Auto & $\mathrm{E}$ & $\mathrm{H}$ \\
\hline \multicolumn{5}{|l|}{ Cannabaceae } \\
\hline Trema micrantha (L.) Blume & $\mathrm{Pi}$ & Zoo & $\mathrm{N}$ & A \\
\hline \multicolumn{5}{|l|}{ Commelinaceae } \\
\hline Commelina diffusa Burm. f. & $\mathrm{Pi}$ & Auto & $\mathrm{N}$ & $\mathrm{H}$ \\
\hline \multicolumn{5}{|l|}{ Cyperaceae } \\
\hline Cyperus difformis $\mathrm{L}$. & $\mathrm{Pi}$ & Ane & $\mathrm{N}$ & $\mathrm{H}$ \\
\hline Cyperus distans L. f. & $\mathrm{Pi}$ & Ane & $\mathrm{N}$ & $\mathrm{H}$ \\
\hline Cyperus rotundus $\mathrm{L}$. & $\mathrm{Pi}$ & Ane & $\mathrm{N}$ & $\mathrm{H}$ \\
\hline Eleocharis elegans (Kunth) Roem. \& Schult. & $\mathrm{Nc}$ & Auto & $\mathrm{N}$ & $\mathrm{H}$ \\
\hline Hypolytrum pungens (Vahl) Kunth & $\mathrm{Nc}$ & Ane & $\mathrm{N}$ & $\mathrm{H}$ \\
\hline \multicolumn{5}{|l|}{ Fabaceae } \\
\hline Glycine wightii (Graham ex Wight \& Arn.) Verdc. & $\mathrm{Nc}$ & $\mathrm{Nc}$ & $\mathrm{E}$ & $\mathrm{T}$ \\
\hline \multicolumn{5}{|l|}{ Lamiaceae } \\
\hline Hyptis suaveolens (L.) Poit. & $\mathrm{Pi}$ & Zoo & $\mathrm{N}$ & $\mathrm{H}$ \\
\hline \multicolumn{5}{|l|}{ Malvaceae } \\
\hline Sida rhombifolia $\mathrm{L}$. & $\mathrm{Si}$ & Auto & $\mathrm{N}$ & $\mathrm{H}$ \\
\hline Triumfetta semitriloba Jacq. & $\mathrm{Nc}$ & Auto & $\mathrm{N}$ & A \\
\hline \multicolumn{5}{|l|}{ Melastomataceae } \\
\hline Clidemia hirta (L.) D. Don & $\mathrm{Si}$ & Zoo & $\mathrm{N}$ & $\mathrm{B}$ \\
\hline Melastomataceae sp. & - & - & - & - \\
\hline \multicolumn{5}{|l|}{ Onagraceae } \\
\hline Ludwigia alternifolia $\mathrm{L}$. & $\mathrm{Nc}$ & $\mathrm{Nc}$ & $\mathrm{E}$ & $\mathrm{H}$ \\
\hline Ludwigia tomentosa (Cambess.) H. Hara & $\mathrm{Pi}$ & Ane & $\mathrm{N}$ & $\mathrm{B}$ \\
\hline \multicolumn{5}{|l|}{ Phyllantaceae } \\
\hline Phyllanthus tenellus Roxb. & $\mathrm{Nc}$ & Auto & $\mathrm{N}$ & $\mathrm{H}$ \\
\hline \multicolumn{5}{|l|}{ Plantaginaceae } \\
\hline Scoparia dulcis L. & $\mathrm{Si}$ & Auto & $\mathrm{N}$ & $\mathrm{H}$ \\
\hline \multicolumn{5}{|l|}{ Poaceae } \\
\hline Brachiaria plantaginea (Link.) Hitchc. & $\mathrm{Pi}$ & Ane & $\mathrm{E}$ & $\mathrm{H}$ \\
\hline Digitaria sanguinalis (L.) Scop. & $\mathrm{Pi}$ & Ane & $\mathrm{N}$ & $\mathrm{H}$ \\
\hline Echinochloa colona (L.) Link & $\mathrm{Pi}$ & Ane & $\mathrm{N}$ & $\mathrm{H}$ \\
\hline Eleusine indica (L.) Gaertn. & $\mathrm{Pi}$ & Ane & $\mathrm{E}$ & $\mathrm{H}$ \\
\hline Paspalum paniculatum $\mathrm{L}$. & $\mathrm{Nc}$ & Auto & $\mathrm{N}$ & $\mathrm{H}$ \\
\hline Brachiaria plantaginea (Link.) Hitchc. & $\mathrm{Pi}$ & Ane & $\mathrm{E}$ & $\mathrm{H}$ \\
\hline \multicolumn{5}{|l|}{ Rosaceae } \\
\hline Rubus rosifolius $\mathrm{Sm}$. & $\mathrm{St}$ & Zoo & $\mathrm{N}$ & $\mathrm{B}$ \\
\hline \multicolumn{5}{|l|}{ Rubiaceae } \\
\hline Ixora gardneriana Benth. & $\mathrm{St}$ & Zoo & $\mathrm{N}$ & A \\
\hline Spermacoce latifolia Aubl. & $\mathrm{Si}$ & Auto & $\mathrm{N}$ & $\mathrm{H}$ \\
\hline
\end{tabular}




\begin{tabular}{|c|c|c|c|c|}
\hline \multicolumn{5}{|l|}{ Solanaceae } \\
\hline Solanum americanum Mill. & $\mathrm{Pi}$ & Zoo & $\mathrm{N}$ & $\mathrm{H}$ \\
\hline Solanum mauritianum Scop. & $\mathrm{Pi}$ & Zoo & $\mathrm{N}$ & A \\
\hline Solanum paniculatum $\mathrm{L}$. & $\mathrm{Pi}$ & Zoo & $\mathrm{N}$ & $\mathrm{B}$ \\
\hline \multicolumn{5}{|l|}{ Urticaceae } \\
\hline Cecropia hololeuca Miq. & $\mathrm{Pi}$ & Zoo & $\mathrm{N}$ & A \\
\hline
\end{tabular}

Se indican las estrategias de regeneración de las especies, tales como pioneras (Pi), secundarias iniciales ( $\mathrm{Si}$ ), secundarias tardías (St); síndrome de dispersión tales como autocóricas (Auto), anemocóricas (Ane), zoocóricas (Zoo); origen como nativas $(\mathrm{N})$ y exóticas $(\mathrm{E})$ y por sus formas de vida, herbáceas $(\mathrm{H})$, arbustos $(\mathrm{B})$ y árboles $(\mathrm{A})$.

Regeneration strategies of species, such as pioneer (Pi), early secondary ( $\mathrm{Si}$ ), late secondary (St) species; dispersal syndrome such as autochoric (Auto), anemochoric (Ane), zoochoric (Zoo); origin between native (N) and exotic (E) and by their forms of life, herbaceous $(\mathrm{H})$, shrubs $(\mathrm{B})$ and trees $(\mathrm{A})$ are indicated.

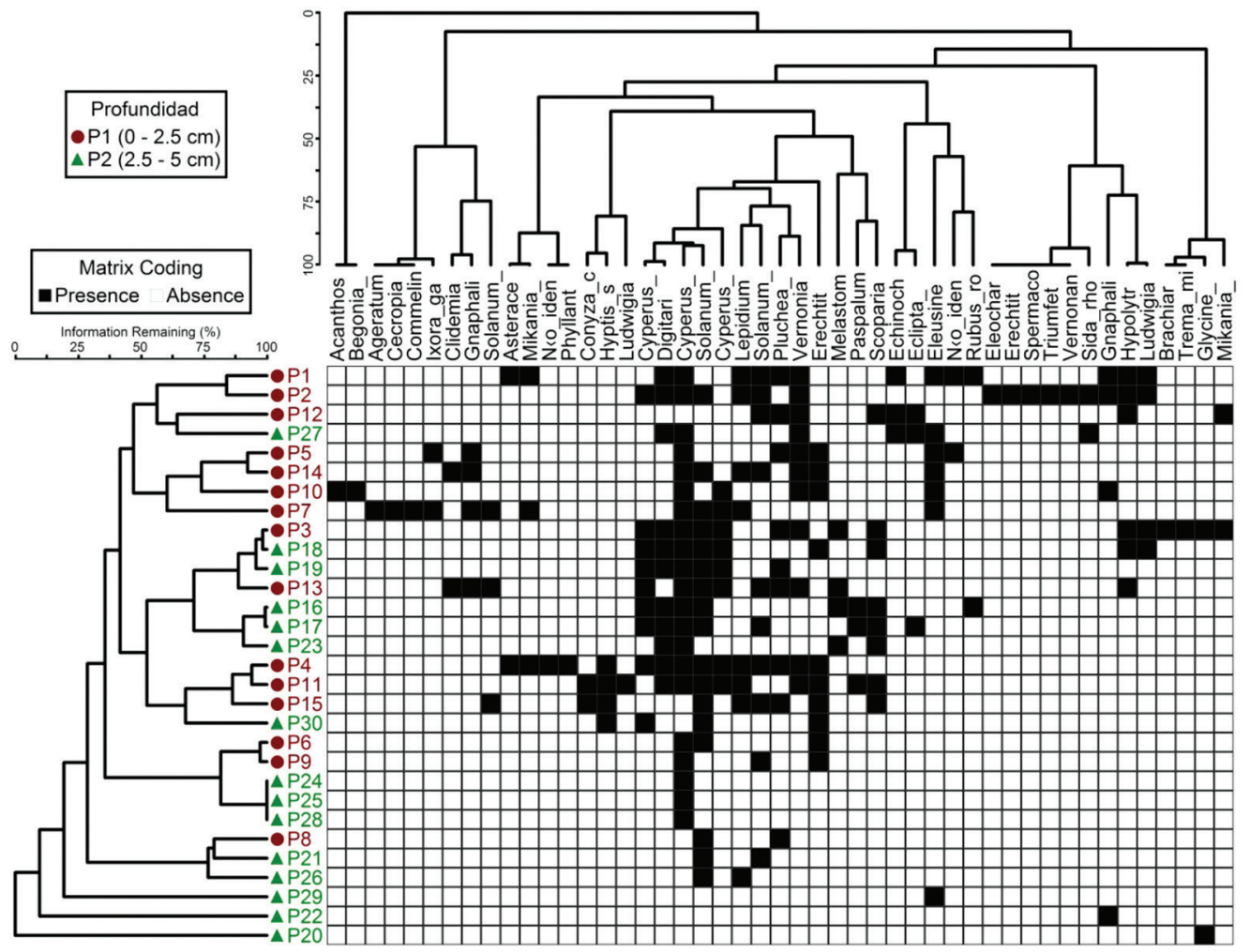

Fig. 3. Dendrograma de conglomerados bidireccional generado en base al índice de disimilitud de Bray-Curtis, que muestra la distribución de 49 especies en dos profundidades de relaves mineros y 30 parcelas en Mariana, Brasil.

Fig. 3. Two-way cluster dendrogram generated based on Sorensen dissimilarity index, which shows the distribution of 49 species at two depths of mining tailings and 30 plots in Mariana, Brazil. 


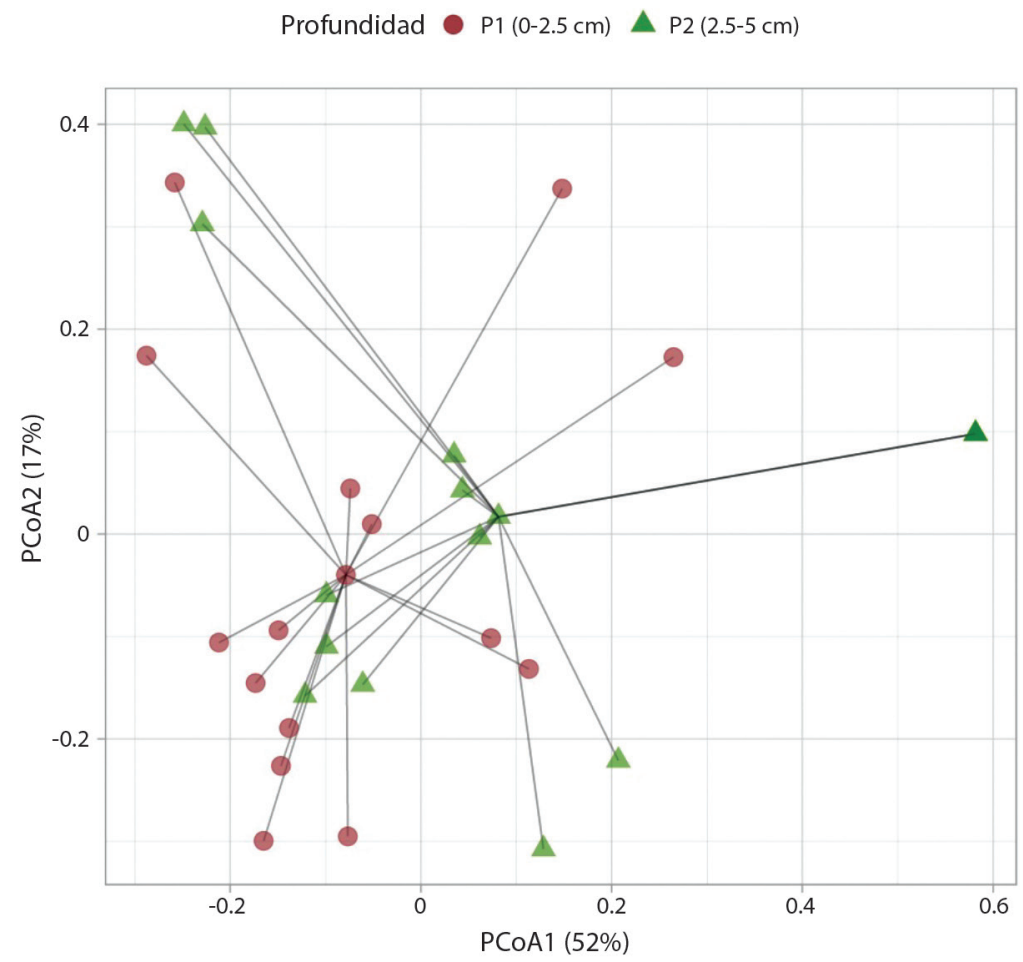

Fig. 4. Análisis de Coordenadas Principales (PCoA) basado en la métrica de disimilitud de Bray-Curtis entre en dos profundidades de relaves mineros en Mariana, Brasil. Se indican las capas superiores $\left(\mathrm{P}_{1}=0-2.5 \mathrm{~cm}\right)$ e inferiores $\left(\mathrm{P}_{2}=\right.$ 2.5-5.0 cm) de relaves.

Fig. 4. Principal Coordinate Analysis (PCoA) based on the Bray-Curtis dissimilarity between the two depths of the mine mudslide in Mariana, Brazil. The upper $\left(\mathrm{P}_{1}=0-2.5 \mathrm{~cm}\right)$ and lower $\left(\mathrm{P}_{2}=2.5-5.0 \mathrm{~cm}\right)$ tailings layers are indicated.

vegetales. La novedad de esta investigación fue observar la marcada diferencia existente entre las dos capas de relaves en términos de riqueza, y estructura (ej. abundancia y densidad de las semillas), a pesar de la poca diferencia de profundidad $(0-5 \mathrm{~cm})$ corroborando la hipótesis propuesta en esta investigación. Se presume que la composición de especies refleja una dispersión reciente el banco de semillas de la capa superior $\mathrm{P}_{1}$, además probablemente podría estar influenciado por la lluvia de semillas de los fragmentos forestales próximos. Por otro lado, el banco de semillas de la capa inferior $\mathrm{P}_{2}$, parece ser resultado de las capas de relaves mineros acumulados. Este patrón de banco de semillas puede tener importantes repercusiones sobre las tendencias futuras de recuperación de la vegetación en el área de estudio. Por este motivo, se discuten y establecen inferencias basadas en investigaciones y experiencias en diferentes escenarios de cambios en el uso de la tierra de paisajes tropicales, principalmente haciendo énfasis en los impactos de actividades mineras.

Diferentes estudios de bosques tropicales reportan que las semillas viables de especies pioneras almacenadas en el suelo pueden desempeñar un papel importante en la rápida regeneración después de los disturbios naturales o antropogénicos (ej. Dalling, Swaine, \& Garwood, 1998; Martins \& Engel, 2007; Mall \& Singh, 2014; Sousa et al., 2017), generalmente depositadas en las primeras capas superficiales del suelo (Baider et al., 2001; Menezes et al., 2019). En este sentido, los resultados de este estudio demuestran un importante 
almacenamiento de semillas viables de especies pioneras en la primera capa del relave, lo que podría indicar su alta calidad y potencial para la regeneración natural. Por el contrario, bancos de semillas de baja calidad con baja densidad de especies pioneras puede retrasar la sucesión secundaria (Wijdeven \& Kuzee, 2000). Este banco de semillas en las capas superficiales del relave en Mariana, representa un importante indicador ecológico clave para la restauración de la vegetación, presentando valores de densidad y riqueza de especies superiores a lo reportado previamente en áreas en recuperación después de actividades de minería (ej. Martins et al., 2008; Miranda-Neto \& Martins, 2017; Miranda-Neto, Martins, \& Silva, 2020). Además, se observó una alta proporción de especies nativas con dispersión anemocórica que representa un conjunto de características funcionales de relevancia para promover la regeneración natural inicial, como ha sido reportado en previos estudios en bosques de la Mata Atlántica (Correia \& Martins, 2015; Balestrin et al., 2019; Miranda-Neto et al., 2020).

Nuestros resultados proporcionan datos de referencia sobre la calidad y potencial del banco de semillas en el área de estudio; es decir, alta riqueza de especies y densidad de semillas, como indicadores clave para seguir monitoreando y llevando a cabo diferentes estrategias de restauración en áreas impactadas por relaves de minería en Mariana. Por un lado, se puede conducir la regeneración natural de acuerdo a los niveles de calidad de los bancos de semillas, y por otro lado, a través de diferentes métodos de restauración activa en áreas de relaves con bancos de semillas de menor calidad y potencial de regeneración. Así mismo, los resultados de alta riqueza de especies y densidad de semillas reportadas en este estudio, permite presumir que es posible aplicar el traslado de semillas colectados en bosques secundarios próximos, los cuales probablemente también son responsables de la alta calidad de los bancos de semillas evaluados. Fue evidente que las áreas impactadas por los efectos de los relaves acumulados, generó la eliminación de la toda la cobertura vegetal existente; sin embargo, en este estudio se demuestra que el potencial de los bancos de semillas para la renovación y el rebrote pueden ser determinantes para la regeneración natural.

A pesar de las diferencias de riqueza, densidad y abundancia de especies entre profundidades, no se observaron diferencias en la composición florística. Sin embargo, las especies dominantes encontradas en los bancos de semillas en áreas afectadas por actividades mineras en paisajes de bosques tropicales han sido principalmente pioneras que promueven una rápida regeneración natural (Miranda-Neto et al., 2020; Martins, Fonseca, Cosimo, \& Balestrin, 2021). Por ejemplo, los resultados indican que la proporción de especies pioneras entre profundidades varía entre 70 y $58 \%$, mientras que de especies secundarias iniciales entre 14 y $23 \%$. Estos grupos de regeneración de rápido crecimiento y exigentes de luz durante la regeneración (Villa et al., 2019), presentan semillas persistentes en el suelo, la mayoría viables para la germinación a largo plazo, hasta cuando se presenten las condiciones favorables para su germinación, principalmente después de un disturbio (Chazdon, 2014; Mall \& Singh, 2014; Bechara et al., 2020; Miranda-Neto et al., 2020). Por el contrario, un menor número de especies secundarias tardías (tolerantes a la sombra) en el banco de semillas, representa un indicador del nivel de disturbio, ya que estas semillas presentan baja viabilidad y alto grado de depredación como principales limitaciones para permanecer en el suelo durante largos periodos de tiempo (Wijdeven \& Kuzee, 2000; Chazdon, 2014). También es posible que exista una limitación de dispersión de semillas de especies secundarias tardías tolerantes a la sombra, y por su característica recalcitrante no ingresan al banco de semillas del suelo, por lo tanto, muchas especies clave con un banco de semillas persistente pueden ser escasas para promover eficazmente la recuperación natural de la vegetación (Török, Helm, Kiehl, Buisson, \& Valkó, 2018; Kildisheva et al., 2020; Mudrák, Řehounková, Vítovcová, Tichý, \& Prach, 2021). La presencia de especies pioneras de árboles como Cecropia hololeuca, Solanum 
mauritianum y Trema micrantha, han sido reconocidas como especies clave que comienzan el proceso de regeneración forestal en áreas degradadas de la región (Martins \& Engel, 2007; Martins, 2009; Miranda-Neto et al., 2020). Por este motivo, se destaca la importancia de estas especies pioneras para acelerar la regeneración natural, así como para establecer condiciones microclimáticas adecuadas para la posterior colonización de especies secundarias tardías (Chazdon, 2014; Martins et al., 2015; Villa et al., 2019).

Los resultados de este estudio muestran que, del total de especies germinadas, se observó un total de 485.4 individuos $\mathrm{m}^{2}$ en $\mathrm{P}_{1}$, y de 82.09 individuos $\mathrm{m}^{2}$ en $\mathrm{P}_{2}$. Las especies pioneras germinan, crecen y se reproducen rápidamente, por lo que su abundancia en el banco de semillas aumenta muy rápidamente (ej. Silva et al. 2019; Martins et al., 2021). Además, es probable que está marcada diferencia entre las dos profundidades de los relaves refleja la importancia de la proximidad de los bosques para enriquecer el banco de semillas, dinámica de transporte y acumulación de relaves, y características funcionales de las semillas (ej. tamaño, peso, síndrome de dispersión), como ha sido repostado en previos estudios (Baider et al., 2001; Menezes et al., 2019; Martins et al., 2021). En este sentido, en bosques tropicales de Panamá se registraron principalmente germinantes de árboles pioneros que tenían entre 330 y 1090 semillas $\mathrm{m}^{2}$ dependiendo de la estación seca y húmeda, respectivamente (Dalling et al., 1997). Por otro lado, en la región de la Mata Atlántica de Brasil, los bosques siempreverdes maduros comprendían entre 389 y 482 semillas viables $\mathrm{m}^{2}$ (Baider et al., 2001). Todos estos estudios de bosques húmedos siempreverdes muestran que existe poca o ninguna asociación entre la composición florística y el banco de semillas, o entre la lluvia anual de semillas y el banco de semillas (Dalling et al., 1998; Mall $\&$ Singh, 2014). Nuestro estudio no fue una excepción, ya que no encontramos especies de árboles de sucesión tardía representadas en el banco de semillas según lo definido por los estudios florísticos de bosques tropicales.
Sin embargo, del total de especies germinadas $89.35 \%$ correspondió a herbáceas, seguido por $7.05 \%$ arbustivas, $0.9 \%$ de especies arbóreas, y $0.3 \%$ de trepadoras o lianas, todas muy importantes en las etapas iniciales de sucesión. Sólo el $2.7 \%$ de las especies registradas en este estudio son de origen exótico, es decir, el banco de semillas de relaves tiene buena calidad para la regeneración natural con especies nativas. Probablemente esto indica que el banco de semillas se recupera muy rápidamente. Sin embargo, es difícil predecir la trayectoria futura del proceso de regeneración de la vegetación.

Con la clasificación de síndromes de dispersión de especies registradas en los bancos de semillas, el $84.12 \%$ de las especies fueron anemocóricas, $9.87 \%$ zoocóricas, y 3.09 $\%$ autocóricas, lo que tiende a coincidir con resultados de otros estudios en bosques de la Mata Atlántica (ej. Baider et al., 2001; Martins et al., 2008; Silva et al., 2019). Por ejemplo, en otros estudios también se ha observado un patrón similar de síndromes de dispersión con especies anemocóricas (entre 50 y $60 \%$ ) y zoocóricas (entre 15 y $30 \%$ ) en bosques tropicales (Chazdon, 2014). De esta forma, el patrón de composición florística observado en los bancos de semillas, principalmente la alta proporción de especies anemocóricas, permite presumir la alta similitud florística entre parcelas y profundidades. Además de la importancia del síndrome de dispersión, dicha agrupación florística puede estar relacionada con ambientes con disturbios a plena exposición solar y sin cobertura vegetal, lo que puede favorecer la llegada de semillas desde los bosques cercanos (Martins et al., 2021; Múdrak et al., 2021). Así mismo, es notable una tendencia hacia un mayor grupo de especies de dispersión herbácea, pionera y anemocórica, que son características notables de este banco de semillas, lo que puede estar induciendo una rápida sucesión secundaria.

En este estudio se determinó que el banco de semillas viables en las capas superficiales de relaves mineros acumulados en el área de estudio, conformados principalmente por especies de origen nativo, pioneras, y anemocóricas, presentan buena calidad y potencial para la 
regeneración natural. Estos resultados demuestran que este patrón de diversidad y características funcionales de las especies (síndromes de dispersión y estrategia de regeneración) pueden ser determinante para promover la regeneración natural de la vegetación en las áreas afectadas con acumulación de relaves mineros en Mariana. Además, este patrón de diversidad y estructura del banco de semillas puede tener importantes repercusiones sobre estrategias de restauración y monitoreo de áreas afectadas por relaves, ya que ambas profundidades de los relaves reflejan la importancia de la proximidad de los bosques para enriquecer el banco de semillas. El enfoque utilizado en nuestro estudio demuestra una base para la distribución de la abundancia y diversidad de especies en la gestión y ecología forestal aplicada, que puede utilizarse para la identificación de especies clave y grupos funcionales que pueden promover la recuperación de los bosques mediante la regeneración natural y restauración activa.

Declaración: los autores declaran que todos están de acuerdo con este artículo y que han hecho aportes que justifican su autoría; que no hay conflicto de interés de ningún tipo; y que han cumplido con todos los requisitos y procedimientos éticos y legales pertinentes para garantizar su publicación.

\section{AGRADECIMIENTOS}

Los autores agradecen al Consejo Nacional de Desarrollo Científico y Tecnológico de Brasil (CNPq), que otorgó becas de investigación para el segundo autor y a la Fundación Renova por el apoyo logístico y para el desarrollo del proyecto. Agradecemos a los revisores anónimos sus importantes comentarios sobre este manuscrito.

\section{RESUMEN}

Introducción: El banco de semillas en el suelo es uno de los indicadores ecológicos más importantes para la evaluación y monitoreo de recuperación de ecosistemas degradados. Así mismo, actualmente indicadores ecológicos de restauración son usados en áreas afectadas por la ruptura de la represa de relaves de Fundão en Mariana, Minas Gerais, Brasil. Objetivo: En este estudio se evaluó la variación de la riqueza, composición de especies, densidad y parámetros fitosociológicos del banco de semillas en dos profundidades de relaves mineros en Mariana, municipio de Mariana, Minas Gerais, Brasil. Métodos: Se recolectaron 15 muestras por nivel de profundidad, en la capa superior (Profundidad1, $\mathrm{P}_{1}=0-2.5 \mathrm{~cm}$ ) y capa inferior (Profundidad2, $\mathrm{P}_{2}=2.5-5.0 \mathrm{~cm}$ ) del relave de minería para examinar el banco de semillas viable, que luego se colocó en un invernadero para la germinación. Posteriormente se contaron e identificaron semanalmente todos los individuos de cada especie durante un período de seis meses. Resultados: Se demostró que existe un banco de semillas en las capas superficiales de relaves mineros acumulados en las áreas afectadas de Mariana, con una marcada diferencia entre profundidades en términos de riqueza de especies y densidad de individuos. Se observó una mayor riqueza y número de individuos en $\mathrm{P}_{1}$, (1 165 semillas germinadas), pertenecientes a 18 familias y 47 especies, en comparación a $\mathrm{P}_{2}$ (197 individuos), pertenecientes a 12 familias y 23 especies. El banco de semillas de la capa $\mathrm{P}_{1}$ está influenciado por la lluvia de semillas del bosque cercano. Por otro lado, el banco de semillas de $\mathrm{P}_{2}$, puede ser característico de los relaves mineros. Conclusiones: El banco de semillas estudiado puede tener efectos positivos sobre la recuperación en el área de estudio, debido a la alta proporción de especies nativas, pioneras y anemocóricas típicas de etapas iniciales de sucesión secundaria.

Palabras clave: disturbios; indicadores ecológicos; estrategia de regeneración; riqueza de especies; síndrome de dispersión.

\section{REFERENCIAS}

Baider, C., Tabarelli, M., \& Mantovani, W. (2001). The soil seed bank during Atlantic Forest regeneration in Southeast Brazil. Revista Brasileira de Biologia, 61(1), 35-44.

Balestrin, D., Martins, S.V., Schoorl, J.M., Lopes, A.T., \& Andrade, C.F. (2019). Phytosociological study to define restoration measures in a mined area in Minas Gerais, Brazil. Ecological Engineering, 135, 8-16.

Bechara, F.C., Salvador, L.Z., Ventura, R.A., Topanotti, L.R., Gerber, D., Cruz, I.S., \& Simonelli, M. (2020). Vegetation and seed bank of an open-scrub bush restinga formation in the Southeastern coast of Brazil. Revista de Biología Tropical, 68(2), 541-550.

Campanharo, I.F., Martins, S.V., Villa, P.M., Kruschewsky, G.C., Dias, A.A., \& Nabeta, F.H. (2020). Effects of forest restoration techniques on community diversity and aboveground biomass on area affected by mining tailings in Mariana, Southeastern Brazil. Research in Ecology, 2, 22-30. 
Campanharo, I.F., Martins, S.V., Villa, P.M., Kruschewsky, G.C., Dias, A.A., \& Nabeta, F.H. (2021). Forest restoration methods, seasonality, and penetration resistance does not influence aboveground biomass stock on mining tailings in Mariana, Brazil. Anais da Academia Brasileira de Ciencias, 93(1), e20201209.

Chao, A., Gotelli, N.J., Hsieh, T.C., Sander, E.L., Ma, K.H., Colwell, R.K., \& Ellison, A.M. (2014). Rarefaction and extrapolation with Hill numbers: a framework for sampling and estimation in species diversity studies. Ecological Monographs, 84(1), 45-67.

Chazdon, R.L. (2014). Second Growth: The Promise of Tropical Forest Regeneration in an Age of Deforestation. Chicago, USA: The University of Chicago Press.

Colwell, R.K., Chao, A., Gotelli, N.J., Lin, S.Y., Mao, C.X., Chazdon, R.L., \& Longino, J.T. (2012). Models and estimators linking individual-based and samplebased rarefaction, extrapolation and comparison of assemblages. Journal of Plant Ecology, 5(1), 3-21.

Correia, G.G.S., \& Martins, S.V. (2015). Banco de sementes do solo de floresta restaurada, Reserva Natural Vale, ES. Floresta e Ambiente, 22(1), 79-87.

Dalling, J.W., Swaine, M.D., \& Garwood, N.C. (1997). Soil seed bank community dynamics in seasonally moist lowland tropical forest, Panama. Journal of Tropical Ecology, 13(5), 659-680.

Dalling, J.W., Swaine, M.D., \& Garwood, N.C. (1998) Dispersal patterns and seed bank dynamics of pioneer trees in moist tropical forest. Ecology, 79(2), $564-578$

Hsieh, T.C., Ma, K.M., \& Chao, A. (2016). 'iNEXT': iNterpolation and EXTrapolation for species diversity (Version 2.0.12, R package). Retrieved from https:// cran.r-project.org/web/packages/iNEXT/iNEXT.pdf

Kildisheva, O.A., Dixon, K.W., Silveira, F.A.O., Chapman, T., Di Sacco, A., Mondoni, A., Turner, S.R., \& Cross, A.T. (2020), Dormancy and germination: making every seed count in restoration. Restoration Ecology, 28(3), S256-S265.

Mall, U., \& Singh, G.S. (2014). Soil seed bank dynamics: history and ecological significance in sustainability of different ecosystems. In M.H. Fulekar, B. Pathak, \& R.K. Kale (Eds.), Environment and sustainable development (pp. 31-46). New Delhi, India: Springer.

Martins, A.M., \& Engel, V.L. (2007). Soil seed banks in tropical forest fragments with different disturbance histories in southeastern Brazil. Ecological Engineering, 31(3), 165-174.

Martins, S.V. (2009). Soil seed bank as indicator of forest regeneration potential in canopy gaps of a semideciduous forest in Southeastern Brazil. In M.V. Fournier (Ed.), Forest regeneration: ecology, management and economics (pp. 113-128). New York, USA: Nova Science Publishers.

Martins, S.V. (2018). Alternative Forest Restoration Techniques. In H. Viana (Ed.), New Perspectives in Forest Science ( $1^{\text {st }}$ ed., pp. 131-148). London, UK: InTech.

Martins, S.V., Almeida, D.P., Fernandes, L.V., \& Ribeiro, T.M. (2008). Banco de sementes como indicador de restauração de uma área degradada por mineração de caulim em Brás Pires, MG. Revista Árvore, 32(6), 1081-1088.

Martins, S.V., Borges, E.E.L., \& Silva, K.A. (2015). O banco de sementes do solo e sua utilização como bioindicador de restauração ecológica. In S.V. Martins (Ed.), Restauração ecológica de ecossistemas degradados ( $2^{\text {nd }}$ ed., pp. 293-330). Viçosa, Brazil: Editora UFV.

Martins, S.V., Fonseca, W.S., Cosimo, L.H.E., \& Balestrin, D. (2021). Soil Seed Banks in Two Environments of Forest Restoration Post Bauxite Mining: Native Tree Plantation and Natural Regeneration. Research in Ecology, 3, 15-25.

Martins, S.V., Villa, P.M., Balestrin, D., Nabeta, F.H., \& Silva, L.F. (2020). Monitoring the passive and active ecological restoration of areas impacted by the Fundão tailings dam disruption in Mariana, Minas Gerais, Brazil. In K. De Vlieger (Ed.), Recent advances in ecological restoration (pp. 83-114). New York, USA: Nova Science Publishers.

Menezes, J.C., Neto, O.C.C., Azevedo, I.F.P., Machado, A.O., \& Nunes, Y.R.F. (2019). Soil Seed Bank at Different Depths and Light Conditions in a Dry Forest in Northern Minas Gerais. Floresta e Ambiente, 26(2), e20170314.

Mirada-Neto, A., \& Martins, S.V. (2017). Banco de sementes em mina de bauxita restaurada no sudeste do Brasil. Floresta e Ambiente, 24, e00125414.

Miranda-Neto, A., Martins, S.V., \& Silva, K.A. (2020). Soil seed banks in different environments: initial forest, mature forest, Pinus and Eucalyptus abandoned stands. Plant Biosystems, 155(1). DOI: $10.1080 / 11263504.2020 .1727979$

Mudrák, O., Řehounková, K., Vítovcová, K., Tichý, L., \& Prach, K. (2021). Ability of plant species to colonise human-disturbed habitats: Role of phylogeny and functional traits. Applied Vegetation Science, 24, e12528.

R Core Team. (2018). R: A language and environment for statistical computing. $R$ Foundation for Statistical Computing. R Foundation for Statistical Computing. Vienna, Austria. Retrieved from https://www.Rproject.org

Silva, K.A., Martins, S.V., Miranda-Neto, A., \& Teixeira, L.A. (2019). Soil Seed Banks in a Forest 
Under Restoration and in a Reference Ecosystem in Southeastern Brazil. Floresta e Ambiente, 26, e20190047. DOI: 10.1590/2179-8087.004719

Silva-Weber, A.J.C., Nogueira, A.C., Carpanezzi, A.A., Galvão, F., \& Weber, S.H. (2012). Composição florística e distribuição sazonal do banco de sementes em Floresta Ombrófila Mista Aluvial, Araucária, PR. Pesquisa Florestal Brasileira, 32(70), 193-207.

Sousa, T.R., Costa, F.R.C., Bentos, T.V., Leal Filho, N., Mesquita, R.C.G., \& Ribeiro, I.O. (2017). The effect of forest fragmentation on the soil seed bank of Central Amazonia. Forest Ecology and Management, 393(1), 105-112.
Török, P., Helm, A., Kiehl, K., Buisson, E., \& Valkó, O. (2018). Beyond the species pool: modification of species dispersal, establishment, and assembly by habitat restoration. Restoration Ecology, 26, S65-S72.

Villa, P.M., Martins, S.V., Rodrigues, A.C., Vieira, N., Bonilla, M.A., \& Ali, A. (2019). Testing species abundance distribution models in tropical forest successions: implications for fine-scale passive restoration. Ecological Engineering, 135, 687-694.

Wijdeven, S.M., \& Kuzee, M.E. (2000). Seed availability as a limiting factor in forest recovery processes in Costa Rica. Restoration Ecology, 8(4), 414-424. 